\title{
DINÂMICA DO CARBONO DA BIOMASSA MICROBIANA EM CINCO ÉPOCAS DO ANO EM DIFERENTES SISTEMAS DE MANEJO DO SOLO NO CERRADO(1)
}

\author{
Eloisa Aparecida Belleza Ferreira ${ }^{(2)}$, Dimas Vital Siqueira Resck ${ }^{(2)}$, \\ Antônio Carlos Gomes ${ }^{(3)} \&$ Maria Lucrécia Gerosa Ramos ${ }^{(4)}$
}

\begin{abstract}
RESUMO
Em um Latossolo Vermelho argiloso, na Embrapa Cerrados, em Planaltina, Distrito Federal, foram selecionadas uma área com vegetação de Cerrado (CE) e seis parcelas de um experimento de longa duração (20 anos): arado de discos préplantio (ADPP); arado de discos pós-colheita (ADPC); plantio direto a partir do segundo ano em área preparada com arado de discos em 1979, primeiro ano de cultivo (PDAD); arado de aivecas pré-plantio (AVPP); arado de aivecas pós-colheita (AVPC); e plantio direto a partir do segundo ano em área preparada com arado de aivecas em 1979, primeiro ano de cultivo (PDAV). Foram estimados o carbono da biomassa microbiana e o carbono orgânico do solo $\left(\mathrm{C}_{\text {solo }}\right)$ em cinco profundidades: 0-5, 5-10, 10-20, 20-30 e 30-40 cm; e em cinco épocas do ano: na época da colheita da soja (abril/2000); no final do período chuvoso, após aração pós-colheita da soja (maio/2000); no período seco, sem nenhuma cultura no campo (agosto/2000); no início do período chuvoso, no plantio do milho (novembro/2000); e na floração da cultura do milho (fevereiro/2001). Considerando todo o período de estudo, os teores de $\mathrm{C}$ da biomassa microbiana $\left(\mathrm{C}_{\text {mic }}\right)$ e de $\mathrm{C}_{\text {solo }}$ foram menores nas camadas mais profundas em todos os sistemas de manejo e no Cerrado. No solo sob sistema plantio direto, os teores de $\mathrm{C}_{\text {mic }}$ e $\mathrm{C}_{\text {solo }}$ decresceram das camadas mais superficiais para as mais profundas e de forma mais acentuada que no solo sob sistemas convencionais. No CE, a biomassa microbiana constituiu-se em maior percentagem
\end{abstract}

\footnotetext{
(1) Parte da Tese de Mestrado em Ciências Agrárias da primeira autora, apresentada à Universidade de Brasília. Recebido para publicação em dezembro de 2002 e aprovado em setembro de 2007.

(2) Pesquisador(a) da Embrapa Cerrados. Km 18, BR 020, Caixa Postal 08223, CEP 73310-970 Planaltina (DF). E-mails: eloisa@cpac.embrapa.br; dvsresck@cpac.embrapa.br

(3) Pesquisador aposentado da Embrapa Cerrados. E-mail: gomes@hotmail.com

(4) Professora da Faculdade de Agronomia e Veterinária, Universidade de Brasília - UnB. Caixa Postal 04508, CEP 70910-900 Brasília (DF). E-mail: lucrecia@unb.br
} 
do carbono orgânico total do solo, em comparação aos sistemas cultivados, que não apresentaram diferenças entre si.

Termos de indexação: carbono orgânico, arado de discos, arado de aivecas, plantio direto, Latossolo, savana.

\title{
SUMMARY: MICROBIAL BIOMASS CARBON DYNAMICS IN DIFFERENT SOIL MANAGEMENT SYSTEMS IN THE CERRADO
}

\begin{abstract}
Microbial biomass carbon and organic carbon were measured in a long-term field experiment (20 years) in Planaltina, DF, Brazil, under corn-soybean crop rotation. Six management systems were selected: disk plow before planting (ADPP); disk plow after harvesting ( $A D P C)$, no-till after disk plowing in the first year (PDAD); moldboard plow before planting (AVPP); moldboard plow after harvesting (AVPC), no-till after moldboard plowing in the first year (PDAV) and an undisturbed area of Savanna type vegetation, Cerrado (CE) as a reference. Soil samples were collected five times a year: 1-April, at soybean harvesting; 2- May, after soybean harvesting; 3-August, in the dry season with no crop on the field; 4- November, in the beginning rainfall season, at corn planting; 5- February, at corn tasseling. The samples were taken from five depths: 0-5, 5-10, 10-20, 20-30 and $30-40 \mathrm{~cm}$ of a clayey Red Latosol (Oxisol). Considering the whole study period, soil microbial carbon $\left(C_{\text {mic }}\right)$ and soil organic carbon $\left(C_{\text {soil }}\right)$ concentration were lower as soil profile depth increased for all management systems as well as in the Cerrado. $C_{\text {mic }}$ and $C_{\text {soil }}$ decreases under no-till system from superficial layers to the deeper ones were more pronounced than in the conventional systems. In the Cerrado, the microbial biomass represented a higher percentage of the total soil organic carbon in comparison to the management systems; there was no difference between conventional and no-till systems.
\end{abstract}

Index terms: organic carbon, disk plow, moldboard plow, no-till, Latosol, Savanna.

\section{INTRODUÇÃO}

Em ecossistemas naturais é mantida estreita e harmônica integração da cobertura vegetal com o sistema físico, químico e biológico do solo, através de processos essenciais, como a ciclagem de nutrientes, pela formação e decomposição da matéria orgânica. Entretanto, essa harmonia é destruída fisicamente, com as operações de preparo do solo, e alterada quimicamente, pela incorporação de corretivos e fertilizantes, causando o desequilíbrio do sistema e, em conseqüência, a aceleração ou retardamento dos processos de ciclagem de nutrientes e de formação e, ou, decomposição da MO (Resck, 1996). Os principais responsáveis por esses processos são os microrganismos do solo (fungos e bactérias), algas e microfauna, cuja massa ou biomassa microbiana está permanentemente em renovação, obtendo a energia da MO, atuando assim como fonte e dreno (mineralização/imobilização temporária) dos nutrientes necessários ao desenvolvimento das plantas. Segundo Jenkinson \& Powlson (1976), a biomassa microbiana está localizada no compartimento lábil da MO, sendo constituída do material orgânico dos microrganismos vivos, contendo entre 1 e $4 \%$ do C total do solo.
Nas últimas três décadas, um grande aumento nas atividades agrícolas ocorreu no Cerrado, e a vegetação nativa foi substituída por áreas cultivadas para produção de alimentos, fibra e energia. Os Latossolos representam $46 \%$ dos solos no Cerrado, com conteúdo médio de MO entre 2,5 e 3,0 dag kg-1 no solo. São solos ácidos, com baixa fertilidade natural, que apresentam boas condições para mecanização; o seu potencial de produção está condicionado ao uso de corretivos e fertilizantes, que necessitam do revolvimento do solo para sua plena distribuição e reação. A mobilização do solo, por proporcionar a incorporação de resíduos orgânicos, pode elevar a biomassa microbiana a curto prazo, por disponibilizar substrato orgânico com a quebra dos agregados, mas, a longo prazo, pode ter efeitos negativos, como a diminuição dos teores de MO (Resck, 1998). Henrot \& Robertson (1994) mediram o C da biomassa microbiana em dois solos tropicais que mostraram padrão similar no declínio da MO e da biomassa microbiana. Após três anos de cultivo convencional, o C total foi reduzido em $20 \%$, e o C microbiano, estabilizado a $35 \%$ do seu valor inicial.

Existem sistemas de manejo que tendem a minimizar o impacto das atividades agrícolas sobre os teores de MO e a estrutura do solo. Desses 
sistemas, destacam-se a pastagem e o plantio direto (PD), que acumulam MO principalmente nos primeiros centímetros do solo (Follet \& Schimel, 1989). Nos sistemas onde o solo é revolvido, a MO é distribuída por toda a camada arável, fazendo com que os teores de carbono orgânico (CO), em profundidades maiores, possam ser semelhantes ou até maiores do que no sistema PD, e isso pode se refletir na atividade e na quantidade da biomassa microbiana (Cattelan \& Vidor, 1990). Em geral, o incremento da biomassa microbiana está relacionado ao aumento do conteúdo de MO do solo (Rezende et al., 2004).

Balota et al. (1996) observaram aumentos de até 129 e $49 \%$ na concentração de $\mathrm{C}$ e $\mathrm{N}$ da biomassa, respectivamente, na camada de $0-5 \mathrm{~cm}$, quando se utilizou plantio direto, comparado com diversos sistemas de cultivo. Carter (1986), trabalhando com trevo forrageiro no Canadá, em solo Charlottetown franco-arenoso, mostrou que, nos primeiros $5 \mathrm{~cm}$ de solo, a biomassa sob plantio direto, por três anos, foi maior que no plantio convencional com arado de aivecas (182 e $111 \mathrm{mg} \mathrm{kg}^{-1}$ de $\mathrm{C}$ no solo, respectivamente); no entanto, entre 5 e $10 \mathrm{~cm}$ de profundidade, o solo sob arado de aivecas apresentou biomassa maior que no PD (247 e $164 \mathrm{mg} \mathrm{kg}^{-1}$ de C no solo, respectivamente).

Vários trabalhos indicam que a distribuição da biomassa no perfil do solo não é uniforme (Oades \& Jenkinson, 1979), sendo conveniente que se quantifique a biomassa em diferentes profundidades. De acordo com Van Gestel et al. (1992), a quantidade de biomassa do solo decresce com a profundidade, independentemente do tempo e sistema de manejo.

Em amostras de 0 a 5 e 25 a $50 \mathrm{~cm}$, no Zimbabwe, em Alfisolos, a biomassa variou de $861 \mathrm{mg} \mathrm{kg}^{-1} \mathrm{de} C$ no solo, na camada superficial, até $233 \mathrm{mg} \mathrm{kg}^{-1} \mathrm{de} \mathrm{C}$ no solo na camada de 25 a $50 \mathrm{~cm}$, em solo sob vegetação nativa (Kirchmann \& Eklund, 1994). Nos solos cultivados durante 15 anos com milho, a biomassa microbiana variou de 397 a $316 \mathrm{mg} \mathrm{kg}^{-1} \mathrm{de}$ $\mathrm{C}$ no solo, nas mesmas profundidades.

Em experimento conduzido por Cerri et al. (1985), foi observado que a biomassa microbiana em Latossolo Amarelo na Amazônia apresentou distribuição mais uniforme nos primeiros $15 \mathrm{~cm}$ do solo, começando a decrescer a partir desta, tornando-se praticamente nula abaixo de $30 \mathrm{~cm}$. Cattelan \& Vidor (1990), no sul do País, analisando somente os $15 \mathrm{~cm}$ superficiais do solo, observaram que os primeiros $5 \mathrm{~cm}$ apresentaram maiores valores de biomassa e atividade microbiana que os da camada de $5-15 \mathrm{~cm}$.

Embora componentes do reservatório de $\mathrm{C}$ tenham diferido entre ecossistemas nativos e cultivados, a maioria dos trabalhos publicados concentra-se em profundidades de até $20 \mathrm{~cm}$ e em ecossistemas que possuem características edafoclimáticas diferentes das do Cerrado, onde existe volume escasso de informações que expliquem as relações específicas entre o impacto das práticas agrícolas nos teores de $\mathrm{C}$ e o da $\mathrm{MO}$ biologicamente ativa, representada pela biomassa microbiana.

Enfoques baseados na caracterização da MO ativa, com tempo de ciclagem mais rápido, têm sido sugeridos como uma medida sensível das mudanças na MO do solo (Sparling, 1992); portanto, a biomassa microbiana pode ser considerada um bom indicador das mudanças induzidas pelo sistema de manejo do solo.

O objetivo deste trabalho foi estudar a influência dos diferentes sistemas de manejo do solo na dinâmica do $\mathrm{C}$ da biomassa microbiana $\left(\mathrm{C}_{\text {mic }}\right)$ e no teor de $\mathrm{CO}$ do solo $\left(\mathrm{C}_{\text {solo }}\right)$, em cinco épocas do ano e cinco profundidades de um Latossolo Vermelho no Cerrado.

\section{MATERIAL E MÉTODOS}

\section{Condições locais e descrição dos tratamentos}

A pesquisa foi desenvolvida no campo experimental da Embrapa Cerrados, localizado em Planaltina (DF), num Latossolo Vermelho argiloso, no período de abril de 2000 a fevereiro de 2001 . O clima local é tropical estacional quente e úmido, com temperatura média variando de 20 a $26^{\circ} \mathrm{C}$ durante o ano. A região apresenta precipitação pluviométrica entre $1.200 \mathrm{e}$ $1.700 \mathrm{~mm}$; o período chuvoso inicia-se em outubro e prolonga-se até abril, com histórico de um período sem chuvas de alguns dias dentro dessa estação, conhecido como veranico (Adámoli et al., 1986).

Foram selecionadas uma área com vegetação típica de Cerrado (CE) e seis parcelas de $1.250 \mathrm{~m}^{2}$ de um experimento de longa duração, instalado no ano de 1979: ADPP - preparo com arado de discos pré-plantio (em que o solo é revolvido a uma profundidade de aproximadamente $25 \mathrm{~cm}$ com arado de discos, apenas uma vez ao ano, no início do período chuvoso, na época do plantio, em outubro/novembro); ADPC - preparo com arado de discos pós-colheita (nesse sistema, o solo é revolvido com arado de discos e os resíduos são incorporados a uma profundidade de aproximadamente $25 \mathrm{~cm}$, duas vezes ao ano, logo após a colheita da cultura, no mês de maio, no final da estação chuvosa e antes do plantio, nos meses de outubro/novembro, no início da estação chuvosa); AVPP - preparo com arado de aivecas pré-plantio (o solo é revolvido com arado de aivecas a uma profundidade de aproximadamente $40 \mathrm{~cm}$, apenas uma vez ao ano, na época do plantio da cultura, em outubro/novembro); AVPC - preparo com arado de aivecas pós-colheita (nesse sistema, o solo é revolvido com arado de aivecas a uma profundidade de aproximadamente $40 \mathrm{~cm}$, duas vezes ao ano, logo após a colheita da cultura, no mês de maio, no final da estação chuvosa e antes do plantio, nos meses de outubro/novembro, no início da estação chuvosa); PDAD - plantio direto a partir do segundo ano em área preparada com arado de discos em 1979, 
primeiro ano de cultivo; e PDAV - plantio direto a partir do segundo ano em área preparada com arado de aivecas em 1979, primeiro ano de cultivo.

Em cada parcela de $1.250 \mathrm{~m}^{2}$ foram estabelecidos três pontos de amostragem, distantes $21 \mathrm{~m}$ entre si, numa diagonal, com os pontos extremos a $6 \mathrm{~m}$ da borda limítrofe da parcela, constituindo-se nas repetições de cada tratamento. Cada amostra foi composta por 10 amostras simples por repetição e por profundidade, coletadas em um raio de um metro e meio em torno de cada ponto central de amostragem.

As coletas das amostras de solo foram feitas em cinco épocas do ano, numa rotação soja/milho (Figura 1), com um trado holandês: na colheita da soja (abril/2000), após aração pós-colheita da soja (maio/ 2000), na seca (agosto/2000), no plantio do milho (novembro/2000) e na floração desta cultura (fevereiro/ 2001); e em cinco profundidades: $0-5,5-10,10-20$, $20-30$ e $30-40 \mathrm{~cm}$. Na época do plantio e floração do milho, o solo foi sempre amostrado nas entrelinhas.

\section{Quantificacão do carbono da biomassa microbiana $\left(C_{\text {mic }}\right)$ e carbono orgânico do solo $\left(\mathbf{C}_{\text {solo }}\right)$}

O C da biomassa microbiana foi estimado pelo método da fumigação-incubação (Jenkinson \& Powlson, 1976), com algumas adaptações. As

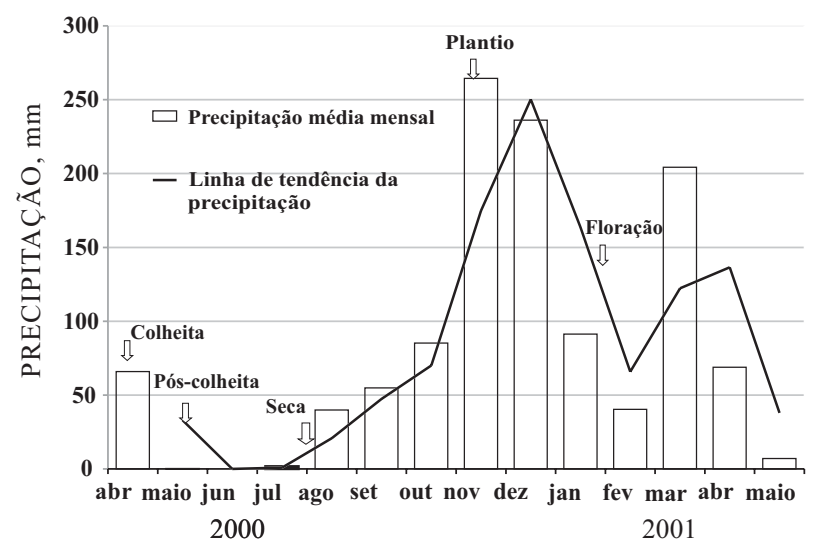

Figura 1. Distribuição de chuvas no período experimental (abril/2000 a maio/2001) e épocas de amostragem. amostras foram armazenadas até o momento das análises em uma câmara fria, com temperatura em torno de $4{ }^{\circ} \mathrm{C}$. Os resíduos da cultura, como raízes e restos vegetais, foram removidos cuidadosamente das amostras, que foram passadas em uma peneira de $8 \mathrm{~mm}$ de malha. Seis porções de $20 \mathrm{~g}$ de solo, cujo conteúdo de água foi ajustado para uma tensão de $30 \mathrm{kPa}$, foram pré-incubadas à temperatura ambiente por sete dias em recipientes fechados de $600 \mathrm{~mL}$. Após esse período, o solo foi fumigado com $\mathrm{CHCl}_{3}$ por $48 \mathrm{~h}$, em triplicatas (equivalentes a três amostras analíticas). Após fumigação, as amostras de solo fumigadas, mais três amostras não-fumigadas, foram incubadas à temperatura ambiente por 10 dias, em recipientes equivalentes aos anteriores. $\mathrm{OCO}_{2}$ liberado (respirado) foi absorvido por uma solução de $\mathrm{KOH}$ $0,3 \mathrm{~mol} \mathrm{~L}^{-1}$ durante o período de incubação e determinado por titulação com $\mathrm{HCl}$, após precipitação do carbonato com $\mathrm{BaCl}_{2} 20 \%$. O C da biomassa microbiana $\left(\mathrm{C}_{\text {mic }}\right.$; expresso em $\mathrm{mg} \mathrm{hg}^{-1} \mathrm{de} \mathrm{C}-\mathrm{CO}_{2}$ no solo) foi calculado dividindo-se o fluxo de $\mathrm{C}-\mathrm{CO}_{2}$ (C$\mathrm{CO}_{2}$ respirado pelo solo fumigado menos o respirado pelo solo não-fumigado) por um fator de conversão igual a 0,41 (Anderson \& Domsch, 1978).

O teor de $\mathrm{CO}$ do solo $\left(\mathrm{C}_{\text {solo }}\right)$ foi avaliado pelo método da oxidação por via úmida (Walkley \& Black, 1934).

\section{Análise estatística}

Utilizou-se o programa denominado "Profile" (Colwell, 1978) para diferenciação estatística dos tratamentos, por meio da análise de variância dos valores dos parâmetros de uma regressão polinomial ortogonal, em que y representa as propriedades do solo $\left(\mathrm{C}_{\text {mic }}\right.$ e $\left.\mathrm{C}_{\text {solo }}\right)$ e x são os valores nas profundidades consideradas nesse estudo. Os parâmetros $\mathrm{p}_{0}, \mathrm{p}_{1}, \mathrm{p}_{2} \mathrm{e} \mathrm{p}_{3}$, independentes entre si, foram estimados para cada repetição amostral, considerando-se os pontos médios nos intervalos de profundidade amostrados, p.ex. $2,5 \mathrm{~cm}(0-5 \mathrm{~cm}), 7,5 \mathrm{~cm}(5-10 \mathrm{~cm}), 15 \mathrm{~cm}(10-20 \mathrm{~cm})$, $25 \mathrm{~cm}(20-30 \mathrm{~cm})$ e $35 \mathrm{~cm}(30-40 \mathrm{~cm})$, e testando esses parâmetros com o teste t a $5 \%$. A significância dos parâmetros das equações ortogonais foi avaliada pelo teste $\mathrm{F}$. $\mathrm{O}$ modelo de regressão polinomial ortogonal $\hat{y}=p_{0}+p_{1} x_{L}+p_{2} x_{Q}+p_{3} x_{C}$ é apresentado no quadro 1 , em que $\mathrm{p}_{0}$ representa o efeito médio dos tratamentos no perfil e os parâmetros da regressão $p_{1}, p_{2}$

\section{Quadro 1. Parâmetros e coeficientes de uma equação de regressão polinomial ortogonal}

Descrição do comportamento

Parâmetros e coeficientes da equação que formam o polinômio

\author{
Tendência zero (efeito médio dos tratamentos) \\ Tendência linear \\ Tendência quadrática \\ Tendência cúbica \\ Equação completa
}

$$
\begin{aligned}
\hat{y} & =p_{0} \\
\hat{y} & =p_{1} x_{L} \\
\hat{y} & =p_{2} x_{Q} \\
\hat{y} & =p_{3} x_{C} \\
\hat{y} & =p_{0}+p_{1} x_{L}+p_{2} x_{Q}+p_{3} x_{C}
\end{aligned}
$$


e $\mathrm{p}_{3}$ representam a quantidade e a direção (sinal) de cada tendência linear, quadrática e cúbica dos teores de $\mathrm{C}_{\text {mic }}$ e de $\mathrm{C}_{\text {solo }}$ em profundidade $(\mathrm{cm})$, respectivamente. $\mathrm{X}_{\mathrm{L}}, \mathrm{X}_{\mathrm{Q}}$ e $\mathrm{X}_{\mathrm{C}}$ referem-se ao coeficiente linear, quadrático e cúbico dos polinômios ortogonais para a interpolação, obtidos por meio de um programa escrito em linguagem Fortran (Quadro 2) (Colwell, 1978).

Utilizou-se também a regressão não-ortogonal $\hat{y}=b_{0}+b_{1} x+b_{2} x^{2}+b_{3} x^{3}$, em que $b_{0}, b_{1}, b_{2}$ e $b_{3}$ são interdependentes e representam os estimadores dos parâmetros para o intercepto e os efeitos linear, quadrático e cúbico, respectivamente, para cálculos estimativos dos valores finais das variáveis $\mathrm{C}_{\text {mic, }} \mathrm{C}_{\text {solo }}$ e da relação $\mathrm{C}_{\text {mic }}$ : $\mathrm{C}_{\text {solo }}$ (expressa em \%) no que se refere à profundidade $\mathrm{x}(\mathrm{cm})$. No presente trabalho, para os modelos ortogonal e não-ortogonal, não houve ajuste significativo em todos os tratamentos, para os efeitos quadrático e cúbico.

Quadro 2. Coeficientes para interpolação de polinômios ortogonais nos diferentes sistemas de manejo

\begin{tabular}{cccc}
\hline \multirow{2}{*}{ Profundidade } & \multicolumn{4}{c}{ Coeficiente de interpolação } \\
\cline { 2 - 4 } & $\mathbf{X}_{\mathbf{L}}$ & $\mathbf{X}_{\mathbf{Q}}$ & $\mathbf{X}_{\mathbf{C}}$ \\
\hline cm & linear & quadrático & cúbico \\
2,5 & $-5,8$ & 19,62093 & $-51,3099$ \\
7,5 & $-3,8$ & $-2,38629$ & 60,80235 \\
15 & $-0,8$ & $-20,3971$ & 41,29568 \\
25 & 3,2 & $-16,4115$ & $-83,0397$ \\
35 & 7,2 & 19,57402 & 32,25171 \\
\hline
\end{tabular}

Para efetuar as comparações entre épocas dentro de cada tratamento e entre tratamentos por profundidade, foi utilizado o teste não-paramétrico de Wilcoxon a $5 \%$ (Conover \& Inan, 1976).

As análises de comparação de médias e as equações de regressão foram realizadas utilizando-se o pacote estatístico SAS (SAS, 1989).

\section{RESULTADOS E DISCUSSÃO}

\section{Comparação do carbono da biomassa microbiana $\left(C_{\text {mic }}\right)$ entre as cinco épocas}

Houve diminuição significativa do $\mathrm{C}_{\text {mic }}$ entre a colheita da soja e a época do plantio do milho nos tratamentos AVPP, AVPC e PDAV, e entre a colheita da soja e a floração do milho nos tratamentos ADPP, ADPC e PDAD (Quadro 3).

Considerando que o método da fumigação-incubação mede a biomassa potencial do solo, verificou-se que na época da colheita da soja os restos culturais abundantes e facilmente mineralizáveis proporcionaram substrato para que a biomassa microbiana pudesse se acumular rapidamente com a acelerada decomposição dos resíduos e expressasse seu potencial, quando condições ideais de temperatura e potencial de água foram fornecidas artificialmente durante os sete dias de pré-incubação. A decomposição da leguminosa (soja) disponibilizou $\mathrm{N}$ para decomposição do estrato herbáceo de gramíneas invasoras, especialmente do gênero Brachiaria.

Segundo Vargas \& Scholles (2000), a presença de leguminosas aumenta a disponibilidade de $\mathrm{N}$ no solo,

Quadro 3. Comparação de médias do carbono da biomassa microbiana entre épocas na profundidade de 0 a $40 \mathrm{~cm}$

\begin{tabular}{|c|c|c|c|c|c|}
\hline \multirow{2}{*}{ Tratamento } & \multicolumn{5}{|c|}{ Época } \\
\hline & $\begin{array}{c}\text { Colheita } \\
\text { (Abril/00) }\end{array}$ & $\begin{array}{c}\text { Pós-colheita } \\
\text { (Maio/00) }\end{array}$ & $\begin{array}{c}\text { Seca } \\
(\text { Agosto/00) }\end{array}$ & $\begin{array}{c}\text { Plantio } \\
\text { (Novembro/00) }\end{array}$ & $\begin{array}{c}\text { Floração } \\
(\text { Fevereiro/01) }\end{array}$ \\
\hline $\mathrm{ADPP}^{(1)}$ & $224,90 \mathrm{a}^{(2)}$ & $221,40 \mathrm{a}$ & 207,02 a & $167,02 \mathrm{ab}$ & $136,77 \mathrm{~b}$ \\
\hline $\mathrm{ADPC}$ & $189,31 \mathrm{a}$ & $184,30 \mathrm{a}$ & $173,80 \mathrm{a}$ & $158,4 \mathrm{ab}$ & $115,6 \mathrm{~b}$ \\
\hline PDAD & 251,74 a & $232,27 \mathrm{a}$ & 228,56 a & $205,96 \mathrm{ab}$ & $149,97 \mathrm{~b}$ \\
\hline AVPP & 225,72 a & $202,30 \mathrm{ab}$ & $198,75 \mathrm{ab}$ & $139,68 \mathrm{bc}$ & $133,15 \mathrm{c}$ \\
\hline AVPC & $204,70 \mathrm{a}$ & $178,63 \mathrm{a}$ & $155,59 \mathrm{a}$ & $120,05 \mathrm{~b}$ & $100,11 \mathrm{~b}$ \\
\hline PDAV & $261,00 \mathrm{a}$ & $232,22 \mathrm{ab}$ & $211,01 \mathrm{ab}$ & $180,20 \mathrm{~b}$ & $178,30 \mathrm{~b}$ \\
\hline $\mathrm{CE}$ & $414,60 \mathrm{a}$ & $393,30 \mathrm{a}$ & $383,70 \mathrm{a}$ & $374,20 \mathrm{a}$ & $350,30 \mathrm{a}$ \\
\hline
\end{tabular}

(1) ADPP: preparo com arado de discos pré-plantio, ADPC: preparo com arado de discos pós-colheita, PDAD: plantio direto a partir do segundo ano em área preparada com arado de discos em 1979, primeiro ano de cultivo, AVPP: preparo com arado de aivecas pré-plantio, AVPC: preparo com arado de aivecas pós-colheita, PDAV: plantio direto a partir do segundo ano em área preparada com arado de aivecas em 1979, primeiro ano de cultivo, CE: Cerrado. ${ }^{(2)}$ Valores seguidos da mesma letra na horizontal não apresentam diferenças significativas entre si pelo teste Wilcoxon a $5 \%$. 
o que, na presença de C, aumenta a atividade microbiana. A partir da época do plantio de milho, ocorrida na segunda metade de novembro, a planta estava em pleno período de desenvolvimento e oferecia pouco material decomponível, além de, possivelmente, competir por nutrientes com os microrganismos do solo. No entanto, para Cheng et al. (1996), quando a cultura de verão está plenamente estabelecida, ela pode estimular a comunidade microbiana, por meio do efeito da rizosfera. Deve-se considerar ainda o fato de que nas épocas do plantio e da floração do milho o solo foi coletado sempre nas entrelinhas, e nas outras épocas, pela impossibilidade de detectar as linhas, a amostragem foi feita aleatoriamente em um raio de um metro e meio em torno do ponto central da amostragem, coletando-se em pontos com maior volume de raízes da soja e plantas invasoras, o que provavelmente propiciou condições para acumulação de biomassa microbiana.

Os menores valores de $\mathrm{C}_{\text {mic }}$ a partir do plantio da cultura de milho poderiam ser justificados pela precipitação de mais de $250 \mathrm{~mm}$ de chuva ocorrida nos meses antecedentes à amostragem de solo, o que deve ter aumentado a mineralização de $\mathrm{C}$ e diminuído a disponibilidade de resíduos para a biomassa microbiana, até mesmo no solo sob Cerrado (Quadro 3), onde a tendência de diminuição do $\mathrm{C}_{\text {mic }}$ foi de $16 \%$, durante o período estudado, embora não significativa. Segundo Moreira \& Siqueira (2002), o tipo de vegetação e as condições ambientais são fatores que determinam a quantidade e a qualidade do material que se deposita no solo, influenciando a heterogeneidade da microbiota e a taxa de decomposição.

Quadro 4. Análise de variância dos parâmetros $\mathbf{p}_{0} \mathbf{e}$ $p_{1}$ da regressão polinomial ortogonal $\hat{y}=p_{0}+p_{1} x_{L}$ $+p_{2} x_{Q}+p_{3} x_{C}$, para carbono da biomassa microbiana ( $\mathrm{mg} \mathrm{kg}^{-1}$ de C-CO $\mathrm{CO}_{2}$ no solo) em sete tratamentos, considerando todo o período de estudo

\begin{tabular}{clc}
\hline \multirow{2}{*}{ Tratamento } & \multicolumn{2}{c}{ Parämetro } \\
\cline { 2 - 3 } & $\mathbf{p}_{0}(* * *)$ & $\mathbf{p}_{1}(* * *)$ \\
\hline ADPP(1) & $191,62 \mathrm{bc}^{(2)}$ & $-9,103 \mathrm{ab}$ \\
ADPC & $164,69 \mathrm{bc}$ & $-4,418 \mathrm{a}$ \\
PDAD & $213,70 \mathrm{~b}$ & $-12,180 \mathrm{~b}$ \\
AVPP & $179,91 \mathrm{bc}$ & $-5,375 \mathrm{a}$ \\
AVPC & $151,59 \mathrm{c}$ & $-4,370 \mathrm{a}$ \\
PDAV & $212,75 \mathrm{bc}$ & $-13,819 \mathrm{~b}$ \\
CE & $383,19 \mathrm{a}$ & $-33,239 \mathrm{c}$
\end{tabular}

(1) Ver quadro 3 para descrição dos tratamentos. ${ }^{(2)}$ Valores seguidos da mesma letra na coluna não apresentam diferenças significativas entre si pelo teste t a $5 \%$. ***: Significativo a $1 \%$ pelo teste $\mathrm{F}$.
Comparação do carbono da biomassa microbiana e do solo em diferentes profundidades

\section{Carbono da biomassa microbiana}

Considerando-se todo o período de estudo (as cinco épocas em conjunto), em relação ao efeito médio do perfil $\left(\mathrm{p}_{0}\right)$, o valor encontrado para o $\mathrm{C}_{\text {mic }}$ no tratamento $\mathrm{CE}$ foi significativamente maior do que o de todos outros tratamentos; nos sistemas agrícolas, houve diferenças significativas apenas entre o PDAD e o AVPC (Quadro 4), comprovando os maiores teores de $\mathrm{C}_{\text {mic }}$ no sistema plantio direto, quando comparado com o sistema convencional com arado de aivecas e duplo revolvimento anual; esses resultados também são encontrados em solos das diferentes regiões do mundo (Alvarez et al., 1997; Balota et al., 1998; Guggenberger et al., 1999).

A variação da biomassa microbiana no perfil é dada pelo estimador do parâmetro $\mathrm{p}_{1}$, que representa a taxa de redução linear com a profundidade $\left(\mathrm{mg} \mathrm{kg}^{-1} \mathrm{de} \mathrm{C}\right.$ no solo por $\mathrm{cm}$ de profundidade). Nesse caso, a maior taxa de redução linear nos sistemas agrícolas ocorreu no tratamento PDAV, apresentado no quadro 4, não sendo diferente dos tratamentos PDAD e ADPP. Entre os demais tratamentos que sofreram algum tipo de revolvimento não houve diferença. $\mathrm{O} \mathrm{CE}$ apresentou a maior taxa de redução linear. Semelhante à estratificação e diminuição da MO com o aumento da profundidade (Resck et al., 1995; Resck, 1997; Corazza et al., 1999), há decréscimo acentuado dos valores de $\mathrm{C}_{\text {mic }}$ nos sistemas sob plantio direto, expresso pelo valor negativo de $\mathrm{p}_{1}$.

Para se calcular o valor da biomassa microbiana nos tratamentos a uma determinada profundidade, multiplica-se o estimador $\mathrm{p}_{1}$ pelo coeficiente de interpolação linear $\mathrm{X}_{\mathrm{L}}$ dessa profundidade (Quadro 2) e soma-se o resultado ao efeito $\mathrm{p}_{0}$.

O modelo de regressão linear de primeira ordem (polinômio não-ortogonal) para todos os tratamentos do $\mathrm{C}_{\text {mic }}$ em função da profundidade, considerando todo o período de estudo, está apresentado na figura 2. Observa-se maior inclinação da reta nos tratamentos PDAD, PDAV e CE. Nesses sistemas, a biomassa microbiana está mais concentrada na superfície do solo (Vargas \& Sholles, 2000), onde há maior quantidade de aporte de substrato, fazendo com que os processos de decomposição do substrato e ciclagem de nutrientes aí ocorram com maior intensidade. Nos outros tratamentos, com exceção do ADPP, a biomassa microbiana é melhor distribuída pelo perfil do solo.

As diferenças entre os tratamentos ocorreram principalmente até a profundidade de $10 \mathrm{~cm}$, abaixo da qual não houve mais diferenças significativas entre os sistemas cultivados, com exceção do CE nas profundidades de 10-20 e 20-30 cm (Quadro 5).

Em termos de valores percentuais, considerando a média ponderada do $\mathrm{C}_{\mathrm{mic}}$ até $40 \mathrm{~cm}$ de profundidade, e adotando o solo não perturbado sob Cerrado (CE) 
como o sistema que representa $100 \%$ do $\mathrm{C}$ da biomassa microbiana original, observa-se um gradiente de redução que vai dos sistemas menos perturbados, representados pelo $\mathrm{PDAD}$ com $59 \%$ e o PDAV com $57 \%$ da biomassa existente no Cerrado, até, na seqüência, o $\mathrm{ADPP}$, com $53 \%$, o AVPP, com $52 \%$, e por fim aqueles sistemas que sofrem duplo revolvimento anual: o ADPC e o AVPC, com 47 e $43 \%$, respectivamente.

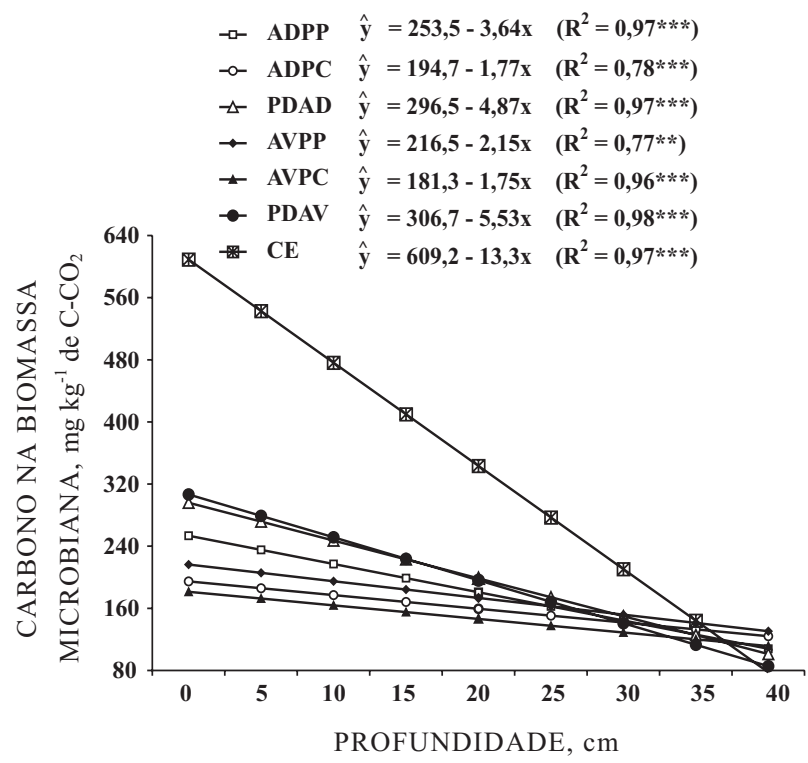

Figura 2. Equações de regressão polinomial nãoortogonal para carbono da biomassa microbiana ( $\hat{y}, \mathrm{mg} \mathrm{kg}^{-1}$ de C-CO $\mathrm{CO}_{2}$ solo) em função da profundidade (cm), considerando todo o período de estudo. $* * *$, **: Significativos a 1 e $5 \%$, respectivamente, pelo teste $F$. Ver quadro 3 para descrição dos tratamentos.
Para os sistemas cultivados, há, em geral, menos $\mathrm{C}$ da biomassa microbiana que no solo sob vegetação nativa. Esses resultados são comparáveis aos encontrados por Renz et al. (1999), em um solo sob vegetação de Cerrado, e aos de Kirchmann e Eklund (1994), que compararam um solo sob savana nativa e um campo adjacente com milho, no Zimbabwe, encontrando menos $\mathrm{C}$ de biomassa microbiana no campo cultivado.

\section{Carbono orgânico do solo $\left(\mathrm{C}_{\text {solo }}\right)$}

Os resultados médios, considerando-se as cinco épocas de amostragem, para valores de $\mathrm{C}_{\text {solo }}$ estão apresentados no quadro 6 (perfil de 0 a $40 \mathrm{~cm}$ de profundidade). Os valores do efeito médio do perfil $\left(\mathrm{p}_{0}\right)$ foram divididos em três grupos distintos: o CE e o PDAD estão no grupo dos maiores valores; em seguida, agrupam-se os tratamentos ADPP, AVPP e PDAV; e o terceiro grupo é composto pelos tratamentos póscolheita (ADPC e AVPC). Os tratamentos em que o solo é revolvido duas vezes ao longo do ano (ADPC e AVPC) tiveram perda de $\mathrm{C}_{\text {solo }}$ maior que a dos outros tratamentos. No ADPC e AVPC, $14 \%$ do $\mathrm{C}_{\text {solo }}$ foi perdido em relação aos tratamentos pré-plantio (ADPP e AVPP). Considerando-se o teor original de $\mathrm{C}$ do solo encontrado no $\mathrm{CE}$ como referência, a perda de $\mathrm{C}_{\text {solo }}$, em ambos os sistemas, seria de $27 \%$. Comparandose o $\mathrm{CE}$ ao $\mathrm{PDAD}$, esse decréscimo no $\mathrm{C}_{\text {solo }}$ foi de $6,3 \%$, o que, em valores absolutos, corresponde, aproximadamente, a 4,5 $\mathrm{t} \mathrm{ha}^{-1}$ de $\mathrm{C}$ perdido, num período de 20 anos, considerando a densidade média do solo no perfil igual a $1,12 \mathrm{~kg} \mathrm{dm}^{-3}$. Segundo Rosa et al. (2003), em cinco anos, tanto o plantio direto de sequeiro com safrinha como o irrigado não foram capazes de restabelecer os níveis de MO original do solo no Cerrado, além de terem causado compactação dos solos estudados.

Quadro 5. Comparação de médias para o carbono da biomassa microbiana em sete tratamentos e em cinco profundidades, considerando todo o período de estudo

\begin{tabular}{|c|c|c|c|c|c|}
\hline \multirow{2}{*}{ Tratamento } & \multicolumn{5}{|c|}{ Profundidade } \\
\hline & $0-5 \mathrm{~cm}$ & $5-10 \mathrm{~cm}$ & $10-20 \mathrm{~cm}$ & $20-30 \mathrm{~cm}$ & $30-40 \mathrm{~cm}$ \\
\hline $\operatorname{ADPP}^{(1)}$ & $238,4 \mathrm{bc}^{(2)}$ & $227,6 \mathrm{bcd}$ & $200,2 \mathrm{ab}$ & $175,9 \mathrm{~b}$ & $116,1 \mathrm{a}$ \\
\hline $\mathrm{ADPC}$ & $176,7 \mathrm{~d}$ & $186,4 \mathrm{~d}$ & $186,8 \mathrm{~b}$ & $144,3 \mathrm{~b}$ & 129,3 a \\
\hline PDAD & $300,2 \mathrm{ab}$ & $247,6 \mathrm{bc}$ & $214,7 \mathrm{ab}$ & $174,8 \mathrm{~b}$ & $131,2 \mathrm{a}$ \\
\hline AVPP & $208,5 \mathrm{~cd}$ & $190,0 \mathrm{~cd}$ & $191,6 \mathrm{~b}$ & $184,7 \mathrm{~b}$ & $124,7 \mathrm{a}$ \\
\hline AVPC & $170,9 \mathrm{~d}$ & $170,6 \mathrm{~d}$ & $160,9 \mathrm{~b}$ & $139,0 \mathrm{~b}$ & $116,1 \mathrm{a}$ \\
\hline PDAV & $284,7 \mathrm{~b}$ & $283,8 \mathrm{ab}$ & $212,6 \mathrm{ab}$ & $166,6 \mathrm{~b}$ & $116,1 \mathrm{a}$ \\
\hline $\mathrm{CE}$ & $609,5 \mathrm{a}$ & $507,2 \mathrm{a}$ & $363,9 \mathrm{a}$ & $266,0 \mathrm{a}$ & $169,4 \mathrm{a}$ \\
\hline
\end{tabular}

\footnotetext{
${ }^{(1)}$ Ver quadro 3 para descrição dos tratamentos. ${ }^{(1)}$ Valores seguidos da mesma letra na coluna não apresentam diferenças significativas entre si pelo teste Wilcoxon a $5 \%$.
} 
Quadro 6. Análise de variância dos parâmetros $p_{0} e$ $p_{1}$ da regressão polinomial ortogonal $\hat{y}=p_{0}+$ $p_{1} x_{L}+p_{2} x_{Q}+p_{3} x_{C}$, para carbono orgânico do solo ( $C_{\text {solo }}$ em dag $\mathrm{kg}^{-1}$ de $C$ no solo) em sete tratamentos, considerando todo o período de estudo

\begin{tabular}{ccc}
\hline \multirow{2}{*}{ Tratamento } & \multicolumn{2}{c}{ Parâmetro } \\
\cline { 2 - 3 } & $\mathbf{p}_{0}(* * *)$ & $\mathbf{p}_{1}(* * *)$ \\
\hline ADPP & $1,49 \mathrm{~b}^{(1)}$ & $-0,042 \mathrm{ab}$ \\
ADPC & $1,29 \mathrm{c}$ & $-0,035 \mathrm{a}$ \\
PDAD & $1,63 \mathrm{a}$ & $-0,060 \mathrm{~cd}$ \\
AVPP & $1,49 \mathrm{~b}$ & $-0,028 \mathrm{a}$ \\
AVPC & $1,27 \mathrm{c}$ & $-0,027 \mathrm{a}$ \\
PDAV & $1,49 \mathrm{~b}$ & $-0,055 \mathrm{bc}$ \\
CE & $1,74 \mathrm{a}$ & $-0,070 \mathrm{~d}$ \\
\hline
\end{tabular}

(1) Ver quadro 3 para descrição dos tratamentos. ${ }^{(2)}$ Valores seguidos da mesma letra na mesma coluna não apresentam diferenças significativas entre si pelo teste t a $5 \%$. ***: Significativo a $1 \%$ pelo teste $\mathrm{F}$.

Alguns dos fatores que contribuem para o declínio da MO são a aceleração dos processos de mineralização devido às operações de preparo do solo, as quais promovem a quebra dos agregados, com subseqüente exposição da MO ao ataque dos microrganismos (Resck et al., 1991), e o aumento de processos erosivos ou baixa adição de $\mathrm{C}$ comparativamente aos sistemas sob vegetação nativa (Angers et al., 1992).

À semelhança da biomassa microbiana, a mais elevada taxa de redução linear do $\mathrm{C}_{\text {solo }}$ com a profundidade $\left(p_{1}\right)$ também ocorreu no $\mathrm{CE}$ - valor significativamente maior que nos outros tratamentos, com exceção do PDAD, indicando que o CO está mais concentrado nas camadas superficiais do solo (Quadro 6). Também não foi observada diferença entre os tratamentos sob PD (média de $\mathrm{p}_{1}=-0,058$ ).

$\mathrm{O} \mathrm{C}$ foi melhor distribuído no perfil do solo nos tratamentos ADPP, ADPC, AVPP e AVPC (Quadro 6), mas houve diminuição do $\mathrm{CO}$ com a profundidade; ainda assim, a maior inclinação da reta é observada nos sistemas plantio direto e no Cerrado em relação aos outros sistemas de manejo (Figura 3), fato confirmado por vários autores (Bayer \& Mielniczuk, 1997; Guedes, 1997).

$\mathrm{O}$ fato de o $\mathrm{C}_{\text {solo }}$ estar mais concentrado nas camadas superficiais dos tratamentos sob sistema plantio direto indica que, no Cerrado, não se pode iniciar o cultivo dos Latossolos e Areias Quartzosas em uma área recém-desbravada sob o sistema plantio direto. Pela grande importância da MO como contribuinte para formação de cargas (mais de $80 \%$ de contribuição na CTC desses solos) e de agregados, a adição de $\mathrm{CO}$ e a sua decomposição em profundidades maiores, além da correção e adubação química, são cruciais para que as plantas cultivadas resistam às intempéries do clima estacional do Cerrado. Essa ação profunda e relativamente rápida é alcançada com uma dinâmica de sistemas de preparo do solo preconizada por Resck (1998), que tem como objetivo condicionar o solo com sistemas convencionais, o mais profundamente possível, química, física e biologicamente, para daí se adotar o cultivo sob o sistema plantio direto.

\section{Relação $\mathbf{C}_{\text {mic }} / \mathbf{C}_{\text {solo }}$ e equações de regressão entre $\mathbf{C}_{\text {mic }}$ e $\mathbf{C}_{\text {solo }}$}

$\mathrm{O}$ quociente entre o $\mathrm{C}$ microbiano $\left(\mathrm{C}_{\text {mic }}\right)$ e o $\mathrm{CO}$ do solo $\left(\mathrm{C}_{\mathrm{solo}}\right)$ é usado para relacionar a biomassa microbiana à disponibilidade do $\mathrm{CO}$ no solo.

Em ecossistemas temperados, a biomassa microbiana representa 0,27 a 5,0 \% do CO total para solos cultivados e 1,8 a 2,9\% para solos de floresta (Anderson \& Domsch, 1986; Vance et al., 1987); em ecossistemas tropicais, esses valores variam de 1,8 a 4 \% (Luizão, 1992; Henrot \& Robertson, 1994).

Em relação ao efeito médio dos tratamentos $\left(\mathrm{p}_{0}\right)$ sobre o CO do solo presente na biomassa microbiana $\left(\mathrm{C}_{\text {mic }} / \mathrm{C}_{\text {solo }}\right)$ em todo o perfil e para o período considerado de estudo, o $\mathrm{CE}$ apresentou valor significativamente maior: $\mathrm{C}_{\text {mic }} / \mathrm{C}_{\text {solo }}=2,12 \%$ (Quadro 7). Não houve diferenças significativas entre os outros tratamentos cujos valores se situaram entre $1,19 \%$ (AVPC) e $1,39 \%$ (PDAV), comparáveis aos valores citados na literatura.

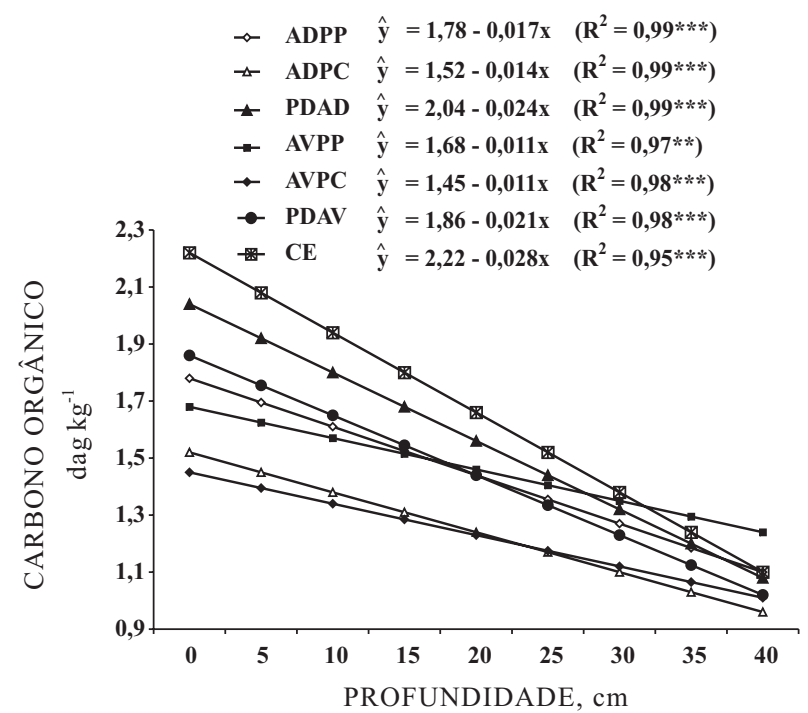

Figura 3. Equações de regressão polinomial nãoortogonal para carbono orgânico $\left(\hat{\mathrm{y}}, \mathrm{dag} \mathbf{~ k g}^{-1}\right.$ solo) em função da profundidade $(\mathrm{cm})$, considerando todo o período de estudo. ***, **: Significativos a 1 e $5 \%$, respectivamente, pelo teste F. Ver quadro 3 para descrição dos tratamentos. 
No presente estudo (Quadro 7), em todos os sistemas a inclinação foi negativa, com exceção do $\mathrm{ADPC}$, demonstrando decréscimo da relação $\mathrm{C}_{\text {mic }} / \mathrm{C}_{\text {solo }}$ com a profundidade, ou seja, diminuição na disponibilidade do CO do solo para a biomassa microbiana em profundidade. No modelo linear nãoortogonal, o ajuste foi significativo nos tratamentos $\mathrm{CE}, \mathrm{PDAD}$ e PDAV, indicando efeito negativo acentuado de profundidade para a relação $\mathrm{C}_{\mathrm{mic}} / \mathrm{C}_{\text {solo }}$ (Quadro 8). Não houve decréscimo significativo da relação $\mathrm{C}_{\text {mic }} / \mathrm{C}_{\text {solo }}$ nos tratamentos sob aração com a profundidade.

Os resultados sugerem que os implementos com arados promovem a distribuição homogênea dos

Quadro 7. Análise de variância dos parâmetros $p_{0} e$ $p_{1}$ da regressão polinomial ortogonal $\hat{y}=p_{0}+p_{1} x_{L}$ $+p_{2} x_{Q}+p_{3} x_{C}$, para a relação $C_{\text {mic }} / C_{\text {solo }}(\%) \mathrm{em}$ sete tratamentos, considerando todo o período de estudo

\begin{tabular}{ccc}
\hline \multirow{2}{*}{ Tratamento } & \multicolumn{2}{c}{ Parâmetro } \\
\cline { 2 - 3 } & $\mathbf{p}_{0}(* * *)$ & $\mathbf{p}_{1}(* * *)$ \\
\hline ADPP $^{(1)}$ & $1,29 \mathrm{~b}^{(2)}$ & $-0,0244 \mathrm{ab}$ \\
ADPC & $1,31 \mathrm{~b}$ & $0,0007 \mathrm{a}$ \\
PDAD & $1,28 \mathrm{~b}$ & $-0,0289 \mathrm{ab}$ \\
AVPP & $1,21 \mathrm{~b}$ & $-0,0148 \mathrm{ab}$ \\
AVPC & $1,19 \mathrm{~b}$ & $-0,0090 \mathrm{ab}$ \\
PDAV & $1,39 \mathrm{~b}$ & $-0,0480 \mathrm{~b}$ \\
CE & $2,12 \mathrm{a}$ & $-0,1165 \mathrm{c}$ \\
\hline
\end{tabular}

${ }^{(1)}$ Ver quadro 3 para descrição dos tratamentos. ${ }^{(2)}$ Valores seguidos da mesma letra na mesma coluna não apresentam diferenças significativas entre si pelo teste t a $5 \%$. ***: Significativo a $1 \%$ pelo teste $\mathrm{F}$. resíduos vegetais e da $\mathrm{MO}$ no perfil do solo como conseqüência do revolvimento, estimulando o metabolismo da biomassa microbiana do solo também nas camadas mais profundas. Segundo Kaiser \& Heinemeyer (1993), o conteúdo de biomassa microbiana de um solo depende, além do conteúdo de $\mathrm{C}$, de vários fatores, como a qualidade e distribuição do $\mathrm{CO}$, que podem sofrer variação espacial e temporal.

Provavelmente, isso tenha ocorrido nos sistemas convencionais estudados, em que houve aumento da quantidade da porção mais humificada e menos disponível da MO em profundidade, com exceção do ADPC. Isso sustentaria a hipótese de Anderson \& Domsh (1986), de que altos valores para essa razão implicam aumento da disponibilidade de substratos mais disponíveis, enquanto razões menores implicam redução dessa disponibilidade. Lavahum et al. (1996) encontraram decréscimos, em cultivo de trigo no inverno, de $2,02 \%$ nos primeiros $10 \mathrm{~cm}$ para $1 \%$ na camada de 30 a $40 \mathrm{~cm}$, e, em solo sob cultivo com espécies de forragem, de $1,31 \%$ para $0,74 \%$ aos $30-$ $40 \mathrm{~cm}$.

Considerando todo o período de estudo, houve relação linear significativa entre o $\mathrm{C}$ da biomassa microbiana $\left(\mathrm{C}_{\mathrm{mic}}\right)$ e o carbono orgâncido do solo $\left(\mathrm{C}_{\text {solo }}\right)$ nos tratamentos: $\mathrm{C}_{\text {mic }} \mathrm{CE}=-235,56+355,50 \mathrm{C}_{\text {solo }}$ $\left(\mathrm{R}^{2}=0,61^{* * *}\right) ; \mathrm{C}_{\text {mic }} \mathrm{PDAD}=-104,83+195,11 \mathrm{C}_{\text {solo }}$ $\left(\mathrm{R}^{2}=0,54^{* * *}\right) ; \mathrm{e} \mathrm{C}_{\mathrm{mic}} \mathrm{PDAV}=-101,980+210,74 \mathrm{C}_{\text {solo }}$ $\left(\mathrm{R}^{2}=0,53^{* * *}\right)$. Isso indica que altos teores de $\mathrm{C}_{\text {solo }}$ do solo podem influenciar positivamente a biomassa microbiana em sistemas conservadores de MO, como o plantio direto e o Cerrado. Guedes (1997) observou, na mesma área experimental do presente estudo, que o sistema plantio direto resultou em mais $\mathrm{C}_{\text {solo }}$ do que o sistema com arado de discos. Esse autor sugeriu que no plantio direto o $\mathrm{CO}$ estaria localizado na fração lábil da MO do solo e, assim, estaria mais disponível como substrato para a biomassa microbiana.

Quadro 8. Equações de regressão polinomial não-ortogonal para a relação $C_{\text {mic }} / \mathbf{C}_{\text {solo }}(\hat{y}$ em percentual em sete tratamentos em função da profundidade, considerando todo o período de estudo

$\begin{array}{lll}\operatorname{ADPP}^{(1)} & \hat{\mathrm{y}}=1,45^{* * *}-0,010^{\mathrm{ns} \mathrm{x}} & \left(\mathrm{R}^{2}=0,73 ; \mathrm{F}=4,8^{* * *}\right) \\ \text { ADPC } & \hat{\mathrm{y}}=1,30^{* * *}-0,0003^{\mathrm{ns} x} & \left(\mathrm{R}^{2}=0,002 ; \mathrm{F}=0,01^{\mathrm{ns}}\right) \\ \text { PDAD } & \hat{\mathrm{y}}=1,48^{* * *}-0,012^{* * * x} & \left(\mathrm{R}^{2}=0,89 ; \mathrm{F}=31,2^{* * *}\right) \\ \text { AVPP } & \hat{\mathrm{y}}=1,31^{* * *}-0,006^{\mathrm{ns} x} & \left(\mathrm{R}^{2}=0,38 ; \mathrm{F}=1,48^{\mathrm{ns}}\right) \\ \text { AVPC } & \hat{\mathrm{y}}=1,25^{* * *}-0,004^{\mathrm{ns} \mathrm{x}} & \left(\mathrm{R}^{2}=0,84 ; \mathrm{F}=1,74^{\mathrm{ns}}\right) \\ \text { PDAV } & \hat{\mathrm{y}}=1,72^{* * *}-0,019^{* * * \mathrm{x}} & \left(\mathrm{R}^{2}=0,93 ; \mathrm{F}=32,15^{* * *}\right) \\ \mathrm{CE} & \hat{\mathrm{y}}=2,91^{* * *}-0,047^{* * * \mathrm{x}} & \left(\mathrm{R}^{2}=0,99 ; \mathrm{F}=77,44^{* * *}\right)\end{array}$

${ }^{(1)}$ Ver quadro 3 para descrição dos tratamentos. ***: Significativo a $1 \%$ e ns: não-significativo pelo teste $\mathrm{F}$. 


\section{CONCLUSÕES}

1. Considerando todo o período de estudo, os teores de $\mathrm{C}$ da biomassa microbiana $\left(\mathrm{C}_{\mathrm{mic}}\right)$ e de $\mathrm{CO}$ do solo $\left(\mathrm{C}_{\text {solo }}\right)$ foram menores nas camadas mais profundas em todos os sistemas de manejo e no Cerrado.

2. No solo sob sistema plantio direto os teores de $\mathrm{C}_{\text {mic }}$ e $\mathrm{C}_{\text {solo }}$ decresceram das camadas mais superficiais para as mais profundas e de forma mais acentuada do que no solo sob sistemas convencionais.

3. No Cerrado, a biomassa microbiana constituiuse em maior percentagem do CO total do solo em comparação com a dos sistemas cultivados, que não apresentaram diferenças entre si.

\section{AGRADECIMENTOS}

À Embrapa Cerrados, pelas condições logísticas e apoio oferecidos, e à FAPDF (Fundação de Apoio à Pesquisa do Distrito Federal), pelo financiamento parcial deste trabalho.

\section{LITERATURA CITADA}

ADÁMOLI, J.; MACEDO, J.; AZEVEDO, L.G. \& MADEIRA NETTO, J. Caracterização da região dos Cerrados. In: GOEDERT, W.J. Solos dos Cerrados: Tecnologias e estratégias de manejo. Planaltina, Embrapa-CPAC/São Paulo, Nobel, 1986. p.33-74.

ALVAREZ, C.R.; ALVAREZ, R.; GRIGERA, M.S. \& LAVADO, R.S. Associations between organic matter fractions and the active soil microbial biomass. Soil Biol. Biochem., 30:767-773, 1997.

ANDERSON, J.P.E. \& DOMSCH, K.H. Mineralization of bacteria and fungi in chloroform-fumigated soils. Soil Biol. Biochem., 10:207-213, 1978.

ANDERSON, T-H. \& DOMSCH, K.H. Carbon assimilation and microbial activity in soil. Z. Pflanzenernachr. Bodenkd., 149:457-468, 1986.

ANGERS, D.A.; PESANT, A. \& VIGNEUX, J. Early croppinginduced changes in soil aggregation, organic matter and microbial Biomass. Soil Sci. Soc. Am. J., 56:115-119, 1992.

BALOTA, E.L.; ANDRADE, D.S. \& COLLOZI-FILHO, A. Avaliações microbiológicas em sistemas de preparo de solo e sucessões de culturas. In: CONGRESSO BRASILEIRO DE PLANTIO DIRETO, 11., Ponta Grossa, 1996. Resumos expandidos. Ponta Grossa, IAPAR-PR, 1996. p.12-14.

BALOTA, E.L.; COLOZZI-FILHO, A.; ANDRADE, D.S. \& HUNGRIA, M.R. Biomassa microbiana e sua atividade em solos sob diferentes sistemas de preparo e sucessão de culturas. R. Bras. Ci. Solo, 22:641-649, 1998.
BAYER, C. \& MIELNICZUK, J. Características químicas do solo afetadas por métodos de preparo e sistemas de cultura. R. Bras. Ci. Solo, 21:105-112, 1997.

CARTER, M.R. Microbial biomasss as an index for tillageinduced changes in soil biological properties. Soil Till. Res., 7:29-40, 1986.

CATTELAN, A.J. \& VIDOR, C. Flutuações na biomassa, atividade da população microbiana do solo, em funções de variações ambientais. R. Bras. Ci. Solo, 14:133-142, 1990.

CERRI, C.C.; VOLKOFF, B. \& EDUARDO, B.P. Efeito do desmatamento sobre a biomassa microbiana em Latossolo amarelo na amazônia. R. Bras. Ci. Solo, 9:1-4, 1985.

CHENG, W.; ZANGH, Q.; COLEMAN, D.C.; CARROL, C.R. \& HOFFMAN, C.A. Is available carbon limiting microbial respiration in rhizosphere? Soil Biol. Biochem., 28:12831288, 1996.

COLWEL, J.D. Computations for studies of soil fertility and fertilizer requirements. Camberra, CSIRO Division of Soils, A.C.T. Australia Commonwealth Agricultural Bureau, 1978. 297p.

CONOVER, W.J. \& INAN, R.L. On some alternative procedures using ranks for the analysis of experimental designes. Comm. Statistics. Part A: Theory Methods, 5:1349-1368, 1976.

CORAZZA, E.J.; SILVA, J.E.; RESCK, D.V.S. \& GOMES, A.C. Comportamento de diferentes sistemas de manejo como fonte ou depósito de carbono em relação à vegetação de Cerrado. R. Bras. Ci. Solo, 23:425-432, 1999.

FOLLET, R.F. \& SCHIMEL, D.S. Effect of tillage practices on microbial biomass dynamics. Soil Sci. Soc. Am. J., 53:10911096, 1989.

GUEDES, H.M. Efeito de diferentes sistemas de manejo na distribuição de classes de agregados e nos teores de carbono orgânico em um Latossolo Vermelho-Escuro argiloso na região dos Cerrados. Brasília, Universidade de Brasília, 1997. 153p. (Tese de Mestrado)

GUGGENBERGER, G.; FREY, S.D.; SIX, J.; PAUSTAN, K. \& ELLIOTT, E.T. Bacterial and fungal cell-wall residues in conventional and no-tillage agroecosystems. Soil Sci. Soc. Am. J., 63:1188-1198, 1999.

HENROT, J. \& ROBERTSON, G.P. Vegetation removal in two soils of the humid tropics: Effect on microbial biomass. Soil Biol. Biochem., 26:111-116, 1994.

JENKINSON, D.S. \& POWLSON, D.S. The effects of biocidal treatments on metabolism in soil. V. A method for measuring soil biomass. Soil Biol. Biochem., 8:209-213, 1976.

KAISER, E.A. \& HEINEMEYER, O. Seasonal variations of soil microbial biomass carbon within the plough layer. Soil Biol. Biochem., 25:1649-1656, 1993. 
KIRCHMANN, H. \& EKLUND, M. Microbial biomass in a savanna-woodland and an adjacent arable soil profile in Zimbabwe. Soil Biol. Biochem., 26:1281, 1994.

LAVAHUM, M.F.E.; JOERGENSEN, R.G. \& MEYER, B. Activity and biomass of soil microorganisms at different depths. Biol. Fert. Soils, 23:38-42, 1996.

LUIZÃO, R.C.C.; BONDE, T.A. \& ROSSWALL, T. Seasonal variation of soil microbial biomass - The effects of clearfelling a tropical rainforest and establishment of pasture in the central Amazon. Soil Biol. Biochem., 24:805813, 1992.

MOREIRA, F.M.S. \& SIQUEIRA, J.O. Microbiologia e bioquímica do solo. Lavras, Universidade Federal de Lavras, 2002. 625p.

OADES, J.M. \& JENKINSON, D.S. Adenoside-triphosphate content of the soil microbial biomass. Soil Biol. Biochem., 11:201-204, 1979.

RENZ, T.E.; NEUFELDT, H.; AYARSA, M.A.; RESCK, D.V.S. \& ZECK, W. Microbial biomass, microbial activity, and carbon pools under different land-use systems in the Brazilian Cerrados. In: THOMAS, R. \& AYARSA, M.A. Dinamics of soil organic matter and indicators of soil quality: Sustanable land manager for the Oxisoils of the Latin America Savanas. Cali, CIAT, 1999. p.187-197.

RESCK, D.V.S. Agricultural intensification systems and their impact on soil and water quality in the Cerrados of Brazil. In: LAL, R. Soil quality and agricultural sustainability. Michigan, Ann Arbor Press, 1998. p.288-300.

RESCK, D.V.S. Manejo de solos e sustentabilidade dos sistemas agrossilvipastoris na região dos Cerrados. In: SIMPÓSIO SOBRE O CERRADO, 8.; INTERNATIONAL SYMPOSIUM ON TROPICAL SAVANNAS, 1., Brasília, 1996. Biodiversidade e produção sustentável de alimentos e fibras nos Cerrados: Anais / Biodiversity and sustainable production of food and fibers in the tropical savannas: Proceedings. Planaltina, Embrapa-CPAC, 1996. p. 81-89.

RESCK, D.V.S. O plantio direto como alternativa de sistema de manejo e conservação do solo e da água na região dos Cerrados. In: CONGRESSO BRASILEIRO DE CIÊNCIA DO SOLO, 26., Rio de Janeiro, 1997. Anais. Rio de Janeiro, Empresa Brasileira de Pesquisa Agropecuária, 1997. CD-ROM.
RESCK, D.V.S.; PEREIRA, J. \& SILVA, J.E. Dinâmica da matéria orgânica na região dos Cerrados. Planaltina, Embrapa-CPAC, 1991. 22p. (Embrapa-CPAC. Documentos, 36)

RESCK, D.V.S. \& SILVA, J.E. Efeito de diferentes sistemas de preparo do solo no teor e na meia-vida da matéria orgânica de um Latossolo Vermelho-Escuro argiloso na região dos Cerrados. In: CONCRESSO BRASILEIRO DE CIÊNCIA DO SOLO, 25., Viçosa, 1995. Resumos. Viçosa, MG, 1995. p.1837-1839.

REZENDE, L.A.; ASSIS, L.C. \& NAHAS, E. Carbon, nitrogen and phosphorous mineralization in two soils amended with distillery yeast. Biores. Technol., 94:159-167, 2004.

ROSA, M.E.C.; OLSZEVSKI, N. \& MENDONÇA, E.S. Formas de carbono em Latossolo Vermelho Eutroférrico sob plantio direto no sistema biogeográfico do Cerrado. R. Bras. Ci. Solo, 27:911-923, 2003.

SAS INSTITUTE. SAS/STAT: User's guide, version 6. 4.ed. Cary, 1989. v.1. 943p.

SPARLING, G.P. Ratio of microbial biomass carbon to soil organic carbon as a sensitive indicator of changes in soil organic matter. Aust. J. Soil. Res., 30:195-207, 1992.

van GESTEL, M.; LADD, J.N. \& AMATO, M. Microbial biomass responses to seasonal change and imposed drying regimes at increasing depths of undisturbed topsoil profiles. Soil Biol. Biochem., 24:103-111, 1992.

VANCE, E.D.; BROOKES, P.C. \& JENKINSON, D.S. An extraction method for measuring soil microbial biomass C. Soil Biol. Biochem., 19:703-707, 1987.

VARGAS, L.K. \& SCHOLLES, D. Biomassa microbiana e produção de $\mathrm{C}-\mathrm{CO}_{2}$ e $\mathrm{N}$ mineral de um Podzólico Vermelho-Escuro submetido a diferentes sistemas de manejo. R. Bras. Ci. Solo, 24:35-42, 2000.

WALKLEY, A. \& BLACK, I.A. An examination of the degtyareff method for determining soil organic matter and proposed modification of the cromic titration method. Soil Sci., 37:29-38, 1934. 